\title{
The eXtendable Solar Array System: A Modular Nanosatellite Power System
}

\author{
Rachel Trabert ${ }^{1}$, Andrew Klesh. ${ }^{2}$, Patrick Senatore ${ }^{3}$, Patrick Martinchek ${ }^{1}$, Daniel Becker ${ }^{1}$, Andrew Chou ${ }^{1}$, \\ Clark Hoffman ${ }^{1}$, Nathan McKay ${ }^{1}$, Jeremy Nash ${ }^{1}$, Jeffery Walters ${ }^{1}$, James Cutler ${ }^{4}$, and Darren McKague ${ }^{5}$ \\ University of Michigan, Ann Arbor, MI,48105
}

The eXtendable Solar Array System (XSAS) is a novel CubeSat solar power technology that uses a deployable array for increased solar panel area and gravity gradient stabilization. This system can be packaged into a 1.5U CubeSat configuration and, once deployed, extends out to a maximum of 2 meters. With this additional surface area, XSAS can provide up to five times the amount of power of standard CubeSat configurations as well as provide gravity gradient stabilization. The current version of XSAS has undergone iterated design and testing. In this paper, testing results are presented including ground testing of the apparatus and microgravity deployment. Initial results are provided of the deployment characteristics through position, acceleration and strain measurements and recommendations are made for CubeSat developers working on other deployables based on these findings.

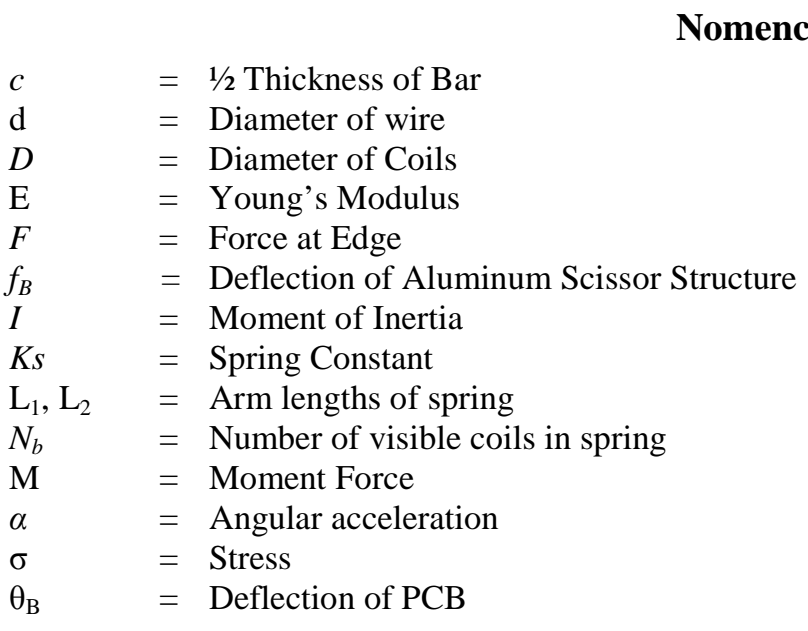

\section{Introduction}

$\mathrm{W}$ ith growing interest in nanosatellites in academic and professional communities, it has become apparent that power, not volume, is the limiting factor for missions using this paradigm. Power availability is dependent on the type of orbit and the inclusion of solar panels or primary batteries, depending on mission needs. With this problem in mind, researchers at the University of Michigan are seeking to increase available power for these satellites. To that end, the eXtendable Solar Array System (XSAS) was designed and constructed by undergraduate and graduate students as part of the Michigan NanoSat Pipeline (MNP) ${ }^{*}$. The

\footnotetext{
${ }^{1}$ Undergraduate Student, University of Michigan Aerospace Engineering, 1320 Beal Ave, Ann Arbor, Mi, 48105, Student Membership.

${ }^{2}$ Postdoctoral Fellow, JAXA Space Exploration Center (JAXA / JSPEC), 3-1-1 Yoshinodai, Sagamihara, Japan, 229-8510, AIAA Member.

${ }^{3}$ Graduate Student, University University of Michigan Aerospace Engineering, 1320 Beal Ave, Ann Arbor, Mi, 48105, Student Membership.

${ }^{4}$ Assistant Professor, University of Michigan Aerospace Engineering, AIAA Member.

${ }^{5}$ Assistant Research Scientist, University of Michigan Atmospheric, Oceanic, and Space Sciences, AIAA Member.

${ }^{*}$ MNP is a program meant to sustain small satellite development at the University of Michigan.
} 
prototype design utilizes 'plug and play' technology to provide a solar power system on a deployable array. In keeping with a modular system, XSAS is a $1.5 \mathrm{U}$ CubeSat that can be integrated into any standard CubeSat configurations. Within its $10 \mathrm{~cm}$ x $10 \mathrm{~cm}$ x $15 \mathrm{~cm}$ package, the full scale XSAS will extend up to 2 meters. When fully extended, XSAS can provide an average of 23 Watts of power, compared to a 3U CubeSat configuration that can provide only 6 Watts $^{1}$. XSAS also has the added benefit of providing gravity gradient stabilization when fully extended. This kind of increased power can expand the number of feasible CubeSat missions by opening the possibilities of instruments to be flown.

XSAS has undergone many design iterations since conception, as shown in Figure 1, with the most current design being discussed in the paper. This paper presents the need for additional testing of XSAS, the computer simulations and predictions of XSAS deployment, the experimental set up for ground and microgravity testing and results and findings from ground and microgravity testing.

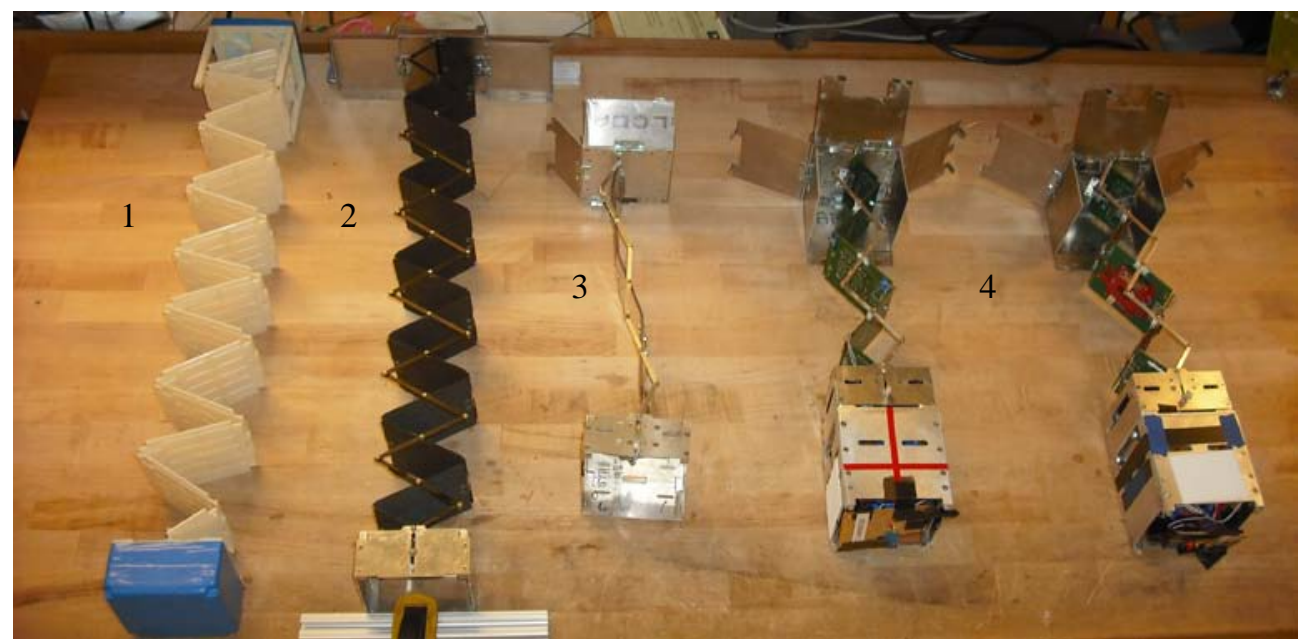

Figure 1. Progression of XSAS Prototypes, 1: First 3D printed model of XSAS, 2: First Full Scale Prototype, 3: Rapid Prototype of microgravity flight model of XSAS, 4: Both microgravity flight models.

\section{Background}

In addition to providing an educational paradigm for young engineers, CubeSats provide an inexpensive platform to test innovative, high-risk technologies. Some of the riskier technologies to be tested are deployable systems. There have been several proposals of deployable systems to be flown on CubeSats since 1999, when the standard was set, though few have successfully flown. One of the most notable CubeSat mission that incorporated deployables was QuakeSat. Launched in 2003, QuakeSat incorporated a telescoping boom and deployable solar panels ${ }^{2}$. The Delfi-C3 mission, quick to follow the example of QuakeSat, successfully incorporated deployable solar panels and antennas ${ }^{3}$.

Due to its strength, robustness, and elastic nature, tapespring is a primary choice for CubeSat deployable mechanisms. Tapespring is the same material found in tape measures and in many construction and carpentry applications ${ }^{4}$. The material has been used to create many different types of structures, such as booms and antennas, and relies on stored potential energy for deployment. The booms that have been flown so far with this type of material do not aid in stabilization of the spacecraft. In the case of QuakeSat, this type of boom was used to isolate a magnetometer from the rest of the spacecraft. There have been a few missions, such as ICE 1 and 2 , that planned to use a $1 \mathrm{~m}$ gravity gradient boom to passively stabilize the CubeSat, but they were unfortunately destroyed upon launch.

There are currently several planned missions that incorporate gravity gradient stabilization. Gravity gradient is a popular choice of passive stabilization since it is easily integrated into the system and does not need to be miniaturized for CubeSats. One of these missions has developed a boom called Gravity-gradient Solar Array Boom, or G-Sab ${ }^{1}$. This boom has similar objectives to XSAS, namely to increase solar panel area and provide gravity gradient stabilization. The boom works by using stored potential energy, with a material similar to tapespring, to unfold the solar panels upon reaching orbit. G-Sab deployment tumbles the CubeSat, requiring long stabilization, whereas XSAS can deploy without inducing additional rotations upon the satellite.

Other types of deployables flown on CubeSats include deployable solar panels that fold up along the sides of the CubeSat as well as deployable antennas. Deployable antennas were flown as early as the first set of CubeSats with AAU CubeSat and QuakeSat. QuakeSat also pioneered deployable solar panels, which were followed by the same type on the Delfi-C3 mission. There are deployment risks associated with any space mechanism. Previous attempts have included satellites such as the MAST mission, which used deployable 
tethers to tie three $1 \mathrm{U}$ CubeSats to each other ${ }^{5}$. Along with a communication failure, the tethers failed to deploy correctly.

These systems unfortunately lack modularity as each was designed for a specific mission. Each satellite program has spent a great deal of time designing their own unique way of deploying an antenna or an additional solar panel. XSAS aims to change this through modularity. Much like companies such as Clyde Space and Pumpkin that sell plug-in parts for CubeSats, XSAS was designed with the thought that it could easily integrate with any 1-1.5U configuration created by the Michigan Nanosat Pipeline and will work for a variety of missions.

It should be noted though that the many moving mechanisms onboard XSAS create significant risk for the space-based system (see Section III A below). This paper will describe the risk mitigation efforts undertaken in the design and deployment of the XSAS system. The current system utilizes a scissor structure with pre-loaded springed hinges to maintain strength before, during and after deployment. Previous research has included the design and construction of a prototype ground-based XSAS hardware model along with a ground-testing rig. This prototype validated the feasibility of the XSAS technology, but additional testing was required for validation in a space-like environment. The microgravity testing results contained in this paper serve to validate existing dynamical models as well as identify unforeseen phenomena that can be accounted for in future higher fidelity models.

\section{Experimental Setup}

Due to the complexity of XSAS and the unique test environment, a great deal of work was completed to design the XSAS structure and establish a robust test plan. Lessons from previous prototypes of XSAS allowed the team to spend time re-designing and optimizing key parts of the XSAS design. Considerations were also given to unique test requirements, such as a quick reset mechanism, that would not be needed in a final flight model. The XSAS design team had three main subgroups to ensure successful ground and microgravity testing: the Payload team (dedicated to working on the XSAS deployable), the Command and Data Handling/Electrical Power System Team, and the Structure Team (dedicated to developing a structure to repeatedly test XSAS for both ground and microgravity environments). Each of these subgroups and their respective projects are described in detail in the following subsections.

\section{A. XSAS Payload Description}

XSAS consists of a 1.5U CubeSat that houses the scissor structure, solar panel array, latching mechanism, and Ni-Chrome deployment mechanism. The full scale model will extend out to 2 meters and provide 23 Watts of power. Due to a size constraint for microgravity testing, though, the model that will be described is a smaller version of XSAS with a total length of $38 \mathrm{~cm}$ fully deployed. From the smaller version a dynamic model will be determined that will be scalable to the full version. As shown in Figure 2, the panels are tilted at a 30 degree angle. This was chosen as the best balance between structural rigidity and incident sun angle for the solar panels. This angle will also be used for the full scale version of XSAS, but can be changed due to mission requirements.
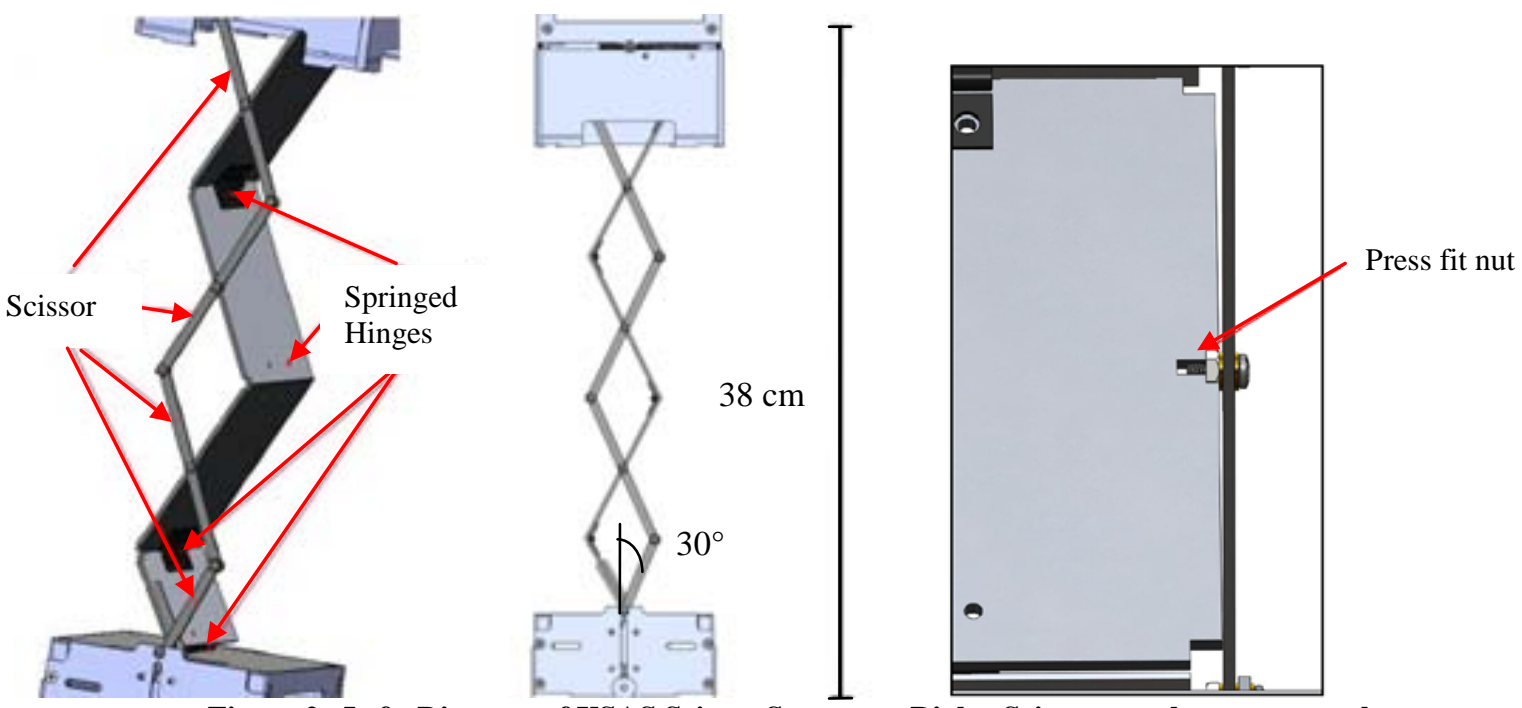

Figure 2. Left: Diagram of XSAS Scissor Structure, Right: Scissor attachment to panel. 
XSAS utilizes a single scissor structure to allow for smooth compression and extension while maintaining stiffness, as seen in Figure 2. The hinges that connect each panel to the rest of the structure have internal torsion springs such that there is a net moment motivating the extension of the scissor structure. The scissor structure is attached to the panels through a single attachment point that allows the scissor and panel to rotate (Figure 2). The force from the springs is largest when XSAS is in its fully compressed configuration, as this corresponds to the largest angular displacement from equilibrium in each of the torsion springs. Four release panels, shown in Figure 3, are attached to each side of the upper assembly by the same type of spring hinges such that they each experience a moment motivating a clearing motion outward and away from the structure. Figure 3 depicts the compression/extension sequence.

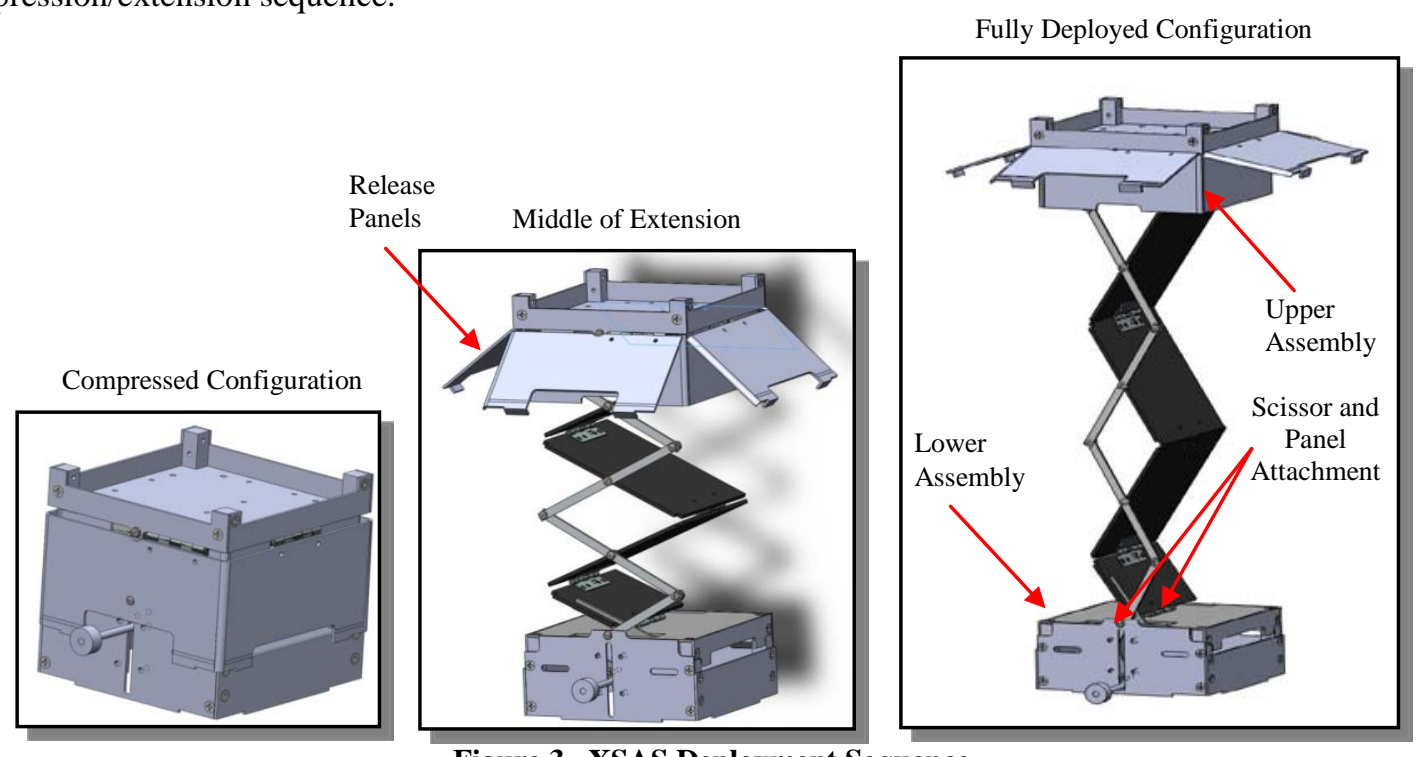

Figure 3. XSAS Deployment Sequence.

The latching mechanism (Figure 4) allows XSAS to lock in place as a rigid structure once it has reached the end of the extension phase. The mechanism is attached to the bottom of the scissor structure on the inside of the front panel. As XSAS extends during deployment, a tab slides down a plate until it reaches an indentation. A spring pushes the tab into the indentation eliminating movement back up the plate and therefore eliminating the movement of the scissor structure. This action corresponds with the time at which XSAS is fully extended. To recompress XSAS, an operator pushes a knob accessible from the outside of the front panel. This knob recompresses the spring, freeing the tab from the indentation. At this point, the scissor structure of XSAS is again free to compress.

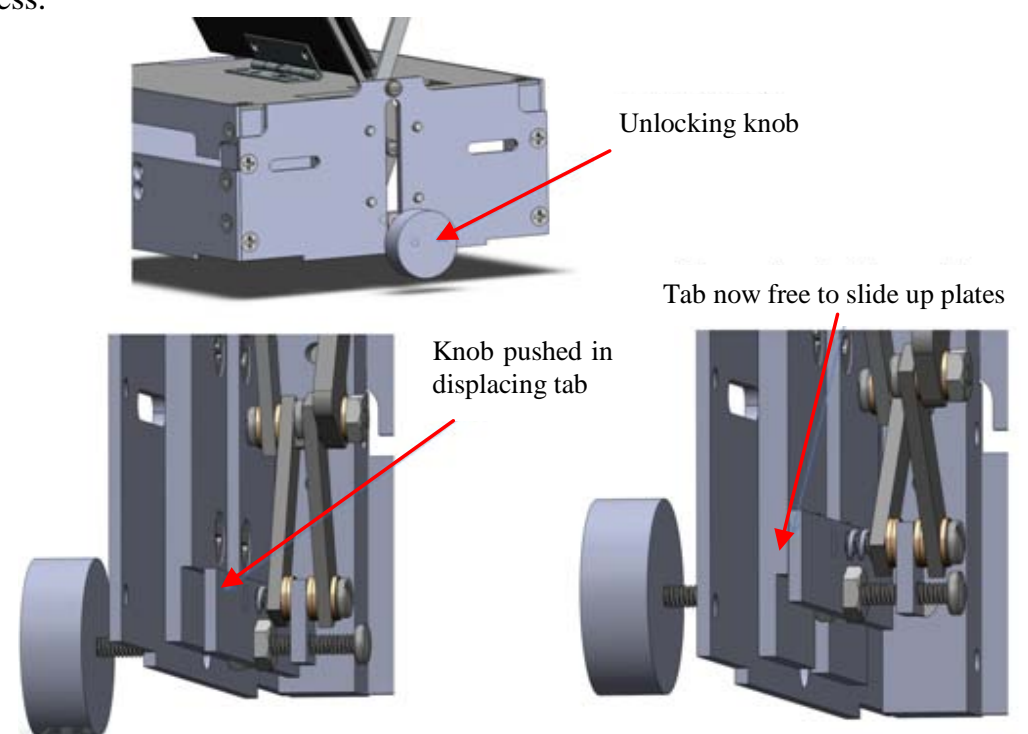

Figure 4. Latching Mechanism locking and release process.

The compression of XSAS is achieved through the compression of the hinge springs. After assuring the latching mechanism is disengaged an operator applies compressive forces on the top and bottom of XSAS so that the scissor structure compresses. Once fully compressed, the operator angularly displaces the spring hinges 
connecting the release panels to the upper assembly until each panel is parallel to its respective side of XSAS. At this time, two feet on each release panel insert into two slots on each side of XSAS as show in Figure 5. An operator then wraps a loop of elastic line around the four sides of XSAS such that the release panels are no longer free to swing outward and away from the structure. In this configuration, the scissor structure cannot extend because the release panel feet inside the slots prevent vertical movement. Elastic string was chosen for microgravity and ground testing because of its ease of application. In the final version Dyneema will be used instead of elastic string because of its strength.
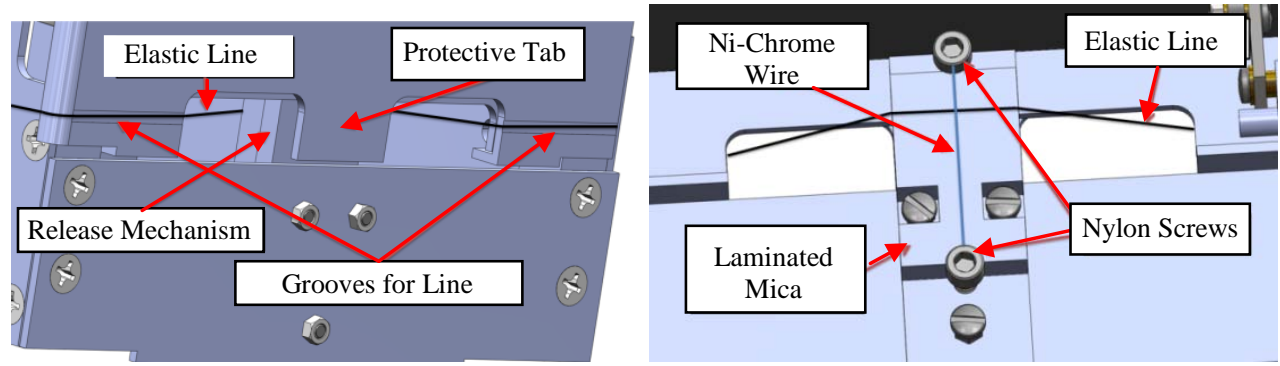

Figure 5. Release Panel Securing Process, Left: Outside of XSAS, Right: Inside of XSAS.

For XSAS to extend from its compressed configuration, the elastic line must be severed. This is accomplished wirelessly. The method employs a Ni-Chrome circuit located on the inside of the lower assembly. When the elastic line is wrapped around XSAS, it is done so such that a portion of the line is in direct contact with the NiChrome. When the wireless signal is sent to supply current to the Ni-Chrome circuit the elastic line is heated for $\sim 400 \mathrm{~ms}$, causing it to break. This eliminates the loop around the release panels allowing them to swing outward releasing their feet from the slots. The scissor structure is then free to extend (see Figure 3). In the full model the deployment will be timed after orbit is reached. For the microgravity application, a wireless signal allowed the team control over when XSAS deployed.

A metal skirt has been added around the solar panels to protect them during launch in the compressed configuration (Figure 6). The skirt serves to transfer launch vibrations through the skin into the attached CubeSat structure. The skirt could also potentially support additional external solar panels. For microgravity and ground testing, solar panels were not used and instead were replaced by identical PCB boards with sensors to help measure the dynamics of XSAS during deployment.

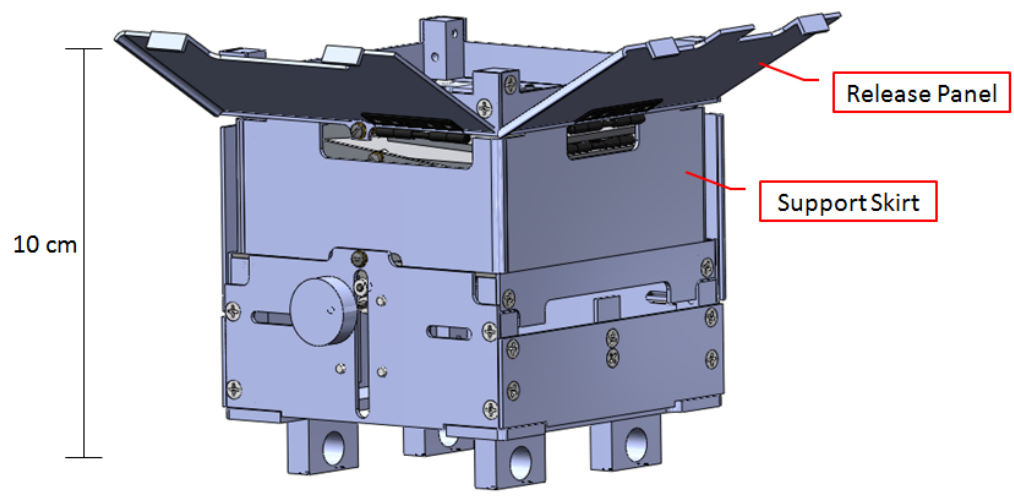

Figure 6. XSAS compressed, showing skirt and deployable panels.

\section{B. Command and Data Handling}

Microgravity testing of XSAS was undertaken to validate and extend existing dynamical models of the deployment. As such, the XSAS panel array features IMUs and strain gauges to measure the acceleration and rotational rates and the bending and torsional forces on the structure, respectively. Dataloggers were used to accurately and consistently record these measurements on each panel and end of the CubeSat.

XSAS uses strain gauge bridges on the each of the expanding panels to the measure torsional and bending forces on the panels during deployment. Each strain gauge bridge is configured in a full bridge configuration to cancel out thermal aberrations, significant drifting, and return an amplified signal to the dataloggers. To measure the bending forces on the panel, four bending strain gauges were placed in the center of each panel, with two on the top side and two on the bottom side as shown in Figure 7. At the conclusion of deployment, these strain gauges face up and down the structure, measuring the bending stresses along the same pathway. To measure the torsional stresses, two torsional strain gauge packages form a full bridge near one side of each 
hinge, with one strain gauge on the top and bottom of the panel at the exact same location. Their purpose is to measure torsional forces that are a result of the hinge interface between the panels. Together, the strain gauges are placed to optimize the amount of stress and strain information received from the panel structures during deployment.
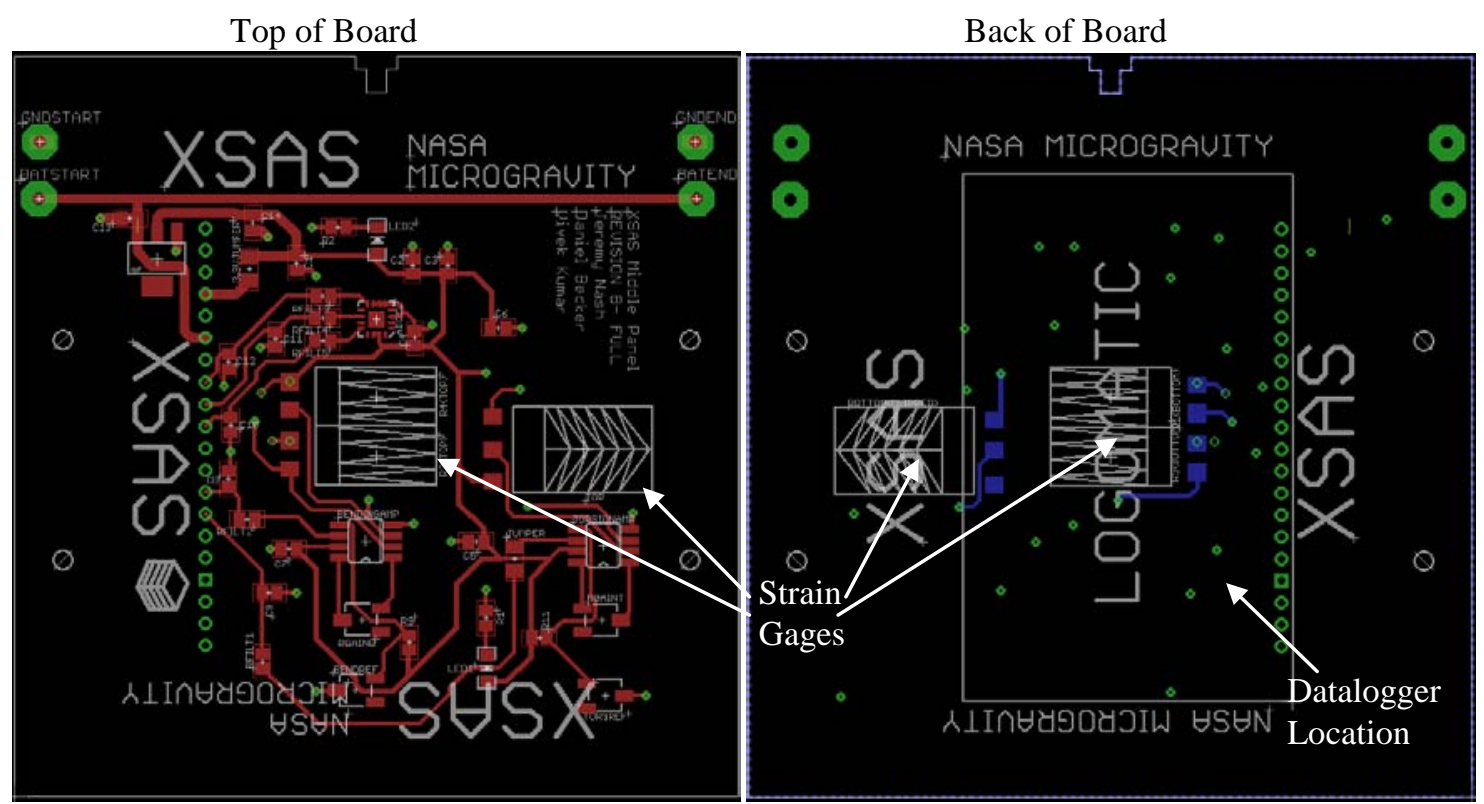

Figure 7. Panel Board Layout for Data Collection.

To monitor XSAS's acceleration and rotational rates during expansion, a six degree of freedom Inertial Measurement Unit (IMU) was used on each end of the satellite. The IMU chosen has a sensitivity and accuracy that matches the force simulations and predictions. Care was taken to note the correspondence in location between the IMUs in order to ascertain the discrepancy in position of the satellite ends in post-processing. The placement of the top IMU is shown in Figure 8. The placement of the bottom IMU is directly underneath XSAS near the latching mechanism.

To measure the analog signals received from the IMUs and strain gauge bridges, a datalogger was used. The datalogger digitizes all of the analog signals and stores them on a microSD card. The same microSD card is used for the entire flight.

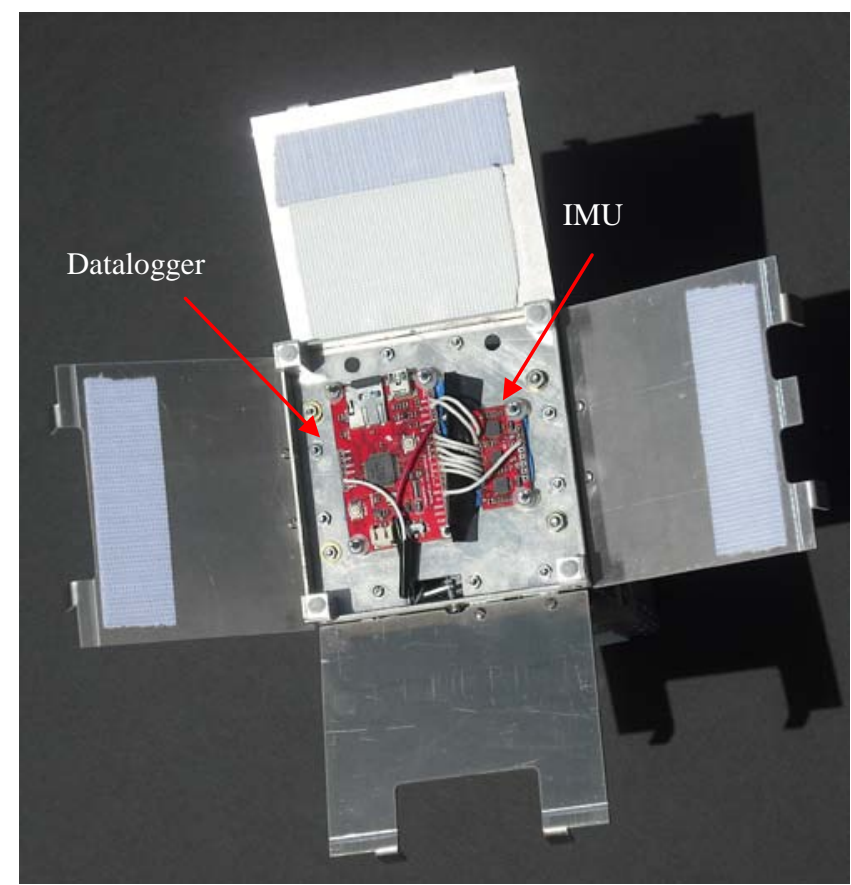

Figure 8. Top IMU and Datalogger. 


\section{Test Structure}

The test structure, shown in Figure 9, holds the necessary equipment to run the experiment and was intended to automate the release of the payload into microgravity. Attached around the structure are handles that can be used by the flyers to control the structure in microgravity. Plastic panels on the outside of the structure encase fragile equipment and protect the fliers from moving parts. The panels also serve as surfaces to secure equipment and supplies for the experiment.

The rear section of the structure is a shelf where the flight laptop and control panel are secured as shown in Figure 9. The control panel contains all the controls needed to automate the release of the payload. The panel also has manual deployment controls to be used if the flight laptop fails. A switch on the side of the control panel activates these back up deployment controls. The backup deployment controls are only activated when needed to prevent accidental deployment commands.

Under the shelf is a platform where the power supply and power strip are attached. The power strip is connected directly to the airplane's power outlets. The power supply and flight laptop are plugged into the power strip. In the event power needs to be immediately cut off, to the power supply or flight laptop, the button on the power strip acts as the emergency power shutoff or "kill" switch. On the platform beside the power supply are strips of Velcro where the primary and backup payloads can be securely stored while not in use. Connected to the two sources of the power supply are wires supplying power to the rotation and release mechanisms.

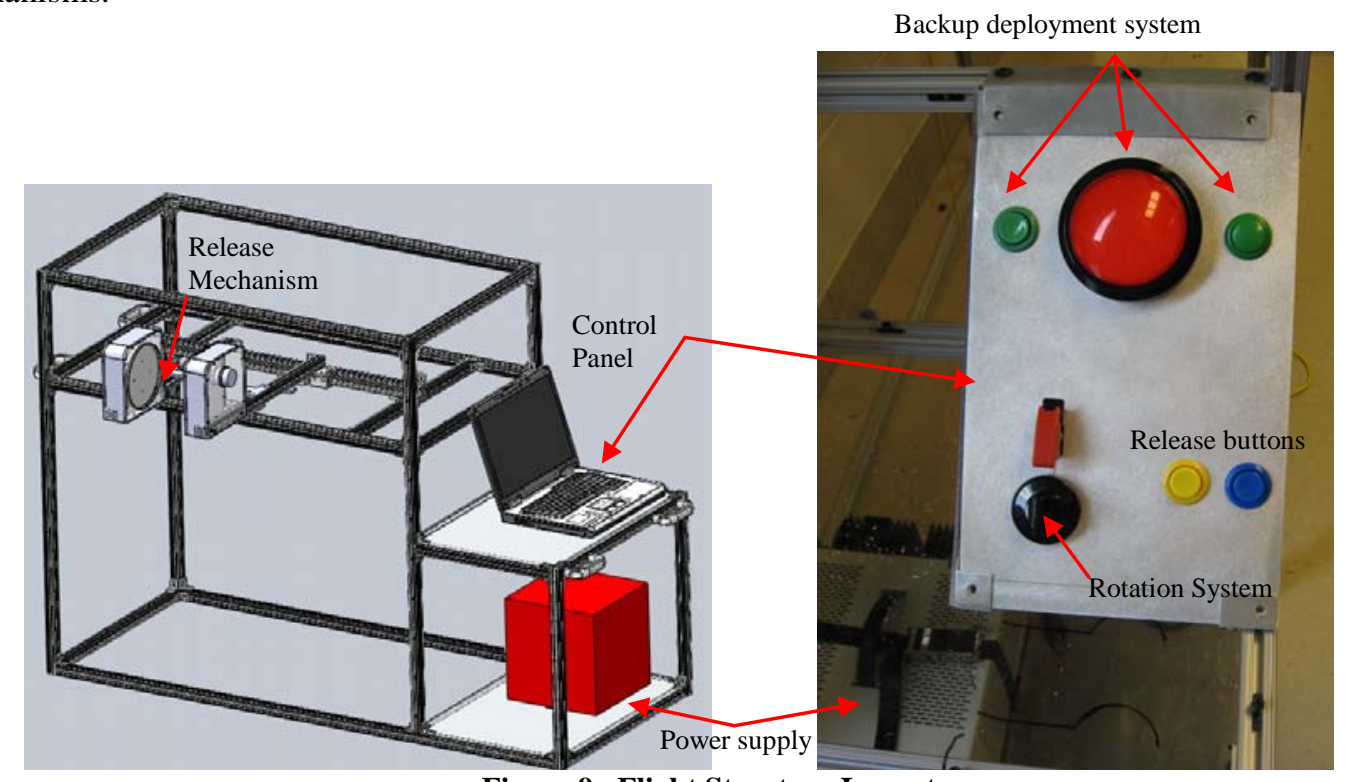

Figure 9. Flight Structure Layout.

The release and rotation mechanisms were intended to automate the release of the payload into microgravity at the rotation rated being evaluated in the experiment. In the latched configuration arms extend from the structure and hold the payload above the ground and away from the structure. These arms are attached to sliders, and bungee cords are used to pull them during automation. For the entire rotation/release/retract sequence a 12V DC motor and two $24 \mathrm{~V}$ pull solenoids are used. Cap screw heads on the feet of the payload fit into holes in the rotating plates on the arms. The payload is sandwiched between these plates and held in place. The plates are on ball bearing turn tables. One plate is also connected to the DC motor. When the appropriate commands are sent via the control panel, the DC motor begins to spin at a calibrated rate. Using gears, the DC motor spins one of the plates holding the payload. The other plate spins in unison as it holds the payload from the other end. When the release button is pushed a solenoid pulls a pin out from a bracket and the arms move apart, releasing the floating payload in the center. When the retract button is pushed a second solenoid pulling a pin causes these arms to move away from the payload and retract into the structure. The payload is now floating freely in front of the structure able to fully extend without hitting anything.

During microgravity testing, the structure did not work as intended to automate the experiment. Unfortunately, the structure was too hard to reset and the payload was released improperly into microgravity. During the first flight day the structure was only used twice before being abandoned. During both release attempts, the payload became stuck to one of the platforms as it was released. It was decided to hand-release the XSAS experiment to obtain additional data rather use flight-time debugging the release mechanism. 


\section{Simulations}

Flight test results were needed to validate dynamical models of XSAS to help predict performance in future development. ND ADAMS software was used to develop this model. The overall XSAS model was first created in Solidworks, and exported to ADAMS using a binary Parasolid format. This initial model as shown in Figure 10 was too complex to simulate, so a reduced model was used in simulations. This reduced model included the solar panel array, scissor structure, and hinges as well as the top and bottom plates to which the array connects. All parts not on the array were replaced with an approximated bulk mass on the top and bottom plates of the assembly (Figure 10).

The characteristics and material properties of the hinge springs that were used to calculate their spring constant were based on Eq. $1-3$, where $N_{b}$ is the number of visible coils, $L_{1}$ and $L_{2}$ are the arm lengths of the spring, $D$ is the diameter of the coils, $d$ is the diameter of the wire, and $E$ is the Young's Modulus of the wire. This spring constant was 0.0238 Newton-meters. Problems encountered during the use of torsion spring constraints on the model prompted us to use a sum of the vertical component forces of all the springs to apply a simple vertical force to the model of 0.04 Newton's. It was predicted that XSAS would deploy in $4.3 \mathrm{sec}$.

$$
\begin{gathered}
N_{e}=\frac{L_{1}+L_{2}}{3 \pi D} \\
N_{a}=N_{b}+N_{e} \\
K S=\frac{d^{4} E}{10.8 D N_{a}}
\end{gathered}
$$
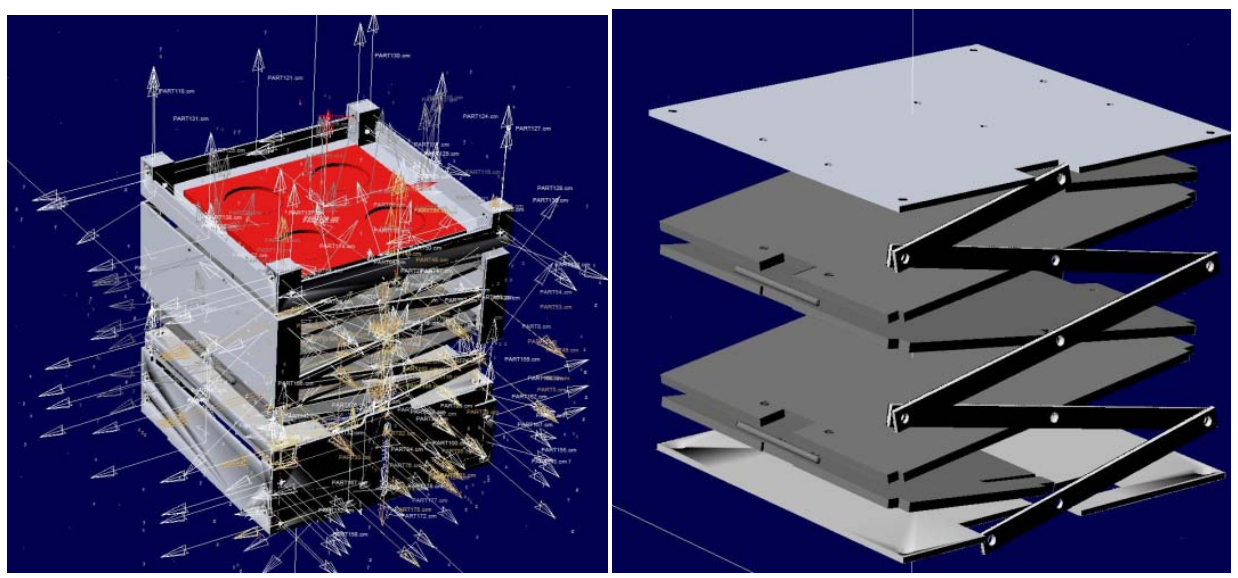

Figure 10. (Left) Full Adams model, (Right) Pared-down XSAS scissor assembly model.

To determine the maximum stress and deflection of the scissor assembly components during deployment and resetting the system was modeled as fully deployed, with the known torsion spring constraints, and a vertical force applied to compress the XSAS model from fully deployed to fully compressed. The vertical compression force was chosen based on a target compression time of 0.5 seconds which, based on the first prototype, is likely a maximum value for resetting XSAS. This resulted in a compression acceleration of $450 \mathrm{~m} / \mathrm{s}^{2}$ applied as a distributed gravity field. From this simulation, the maximum angular acceleration of all the scissor section components was $3927 \mathrm{rad} / \mathrm{s}^{2}$. Using Eq. $4-5$ ( $M$ is the moment force or torque on the element, $c$ is $1 / 2$ the thickness of the bar normal to the torque, $I$ is the moment of inertia of the bar about the torque axis, and $\alpha$ is the angular acceleration), this value along with the characteristics of the PCB plates and Aluminum scissor components, was converted into a stress value on both materials. The calculated maximum stress on the PCB sections is $3.92 \mathrm{~Pa}$, and likewise for the Aluminum bars was $8.81 \mathrm{~Pa}$. This is well below the yield stress values of $300 \mathrm{MPa}$ and $50 \mathrm{MPa}$ respectively.

$$
\begin{aligned}
& M=\propto I \\
& \sigma=\frac{M c}{I}
\end{aligned}
$$


Deflection of the sections was calculated using Eq. $6-8$, where $M$ is the torque on the element, $L$ is the arm length from the torque axis to the edge of the element, $F$ is the derived force at this edge, $E$ is the Young's Modulus of the material, and $I$ is the moment of inertia of the element about the torque axis. The maximum deflection for the PCB is $2.67 \mathrm{e}-10 \mathrm{~mm}$, and $9 \mathrm{e}-12$ radians. The maximum deflection for the aluminum is $9.94 \mathrm{e}-$ $11 \mathrm{~mm}$, and $3 \mathrm{e}-12$ radians.

$$
\begin{gathered}
F=M L \\
\theta_{B}=\frac{F L^{2}}{2 E I} \\
f_{B}=\frac{F L^{3}}{3 E I}
\end{gathered}
$$

\section{Results}

This section will present the results of XSAS testing on the ground and in microgravity. Extensive testing and design work went into developing XSAS. Since this is the first iteration of the XSAS prototype flight model, most of the data is the first that has been recorded and many design iterations are still needed.

\section{A. Ground Tests}

Before the microgravity flight, several tests were performed in the lab. The term 'ground' tests comes from the distinction that these tests were run in 1-g, instead of 0 -g. The purpose of ground testing was to validate the design and provide initial predictions for flight performance. Testing and calibration included: timed deployment tests of XSAS, testing to determine the resonance frequency of XSAS in its deployed configuration, calibration of the bending and torsional strain gauges on the PCB panels, and IMU calibration.

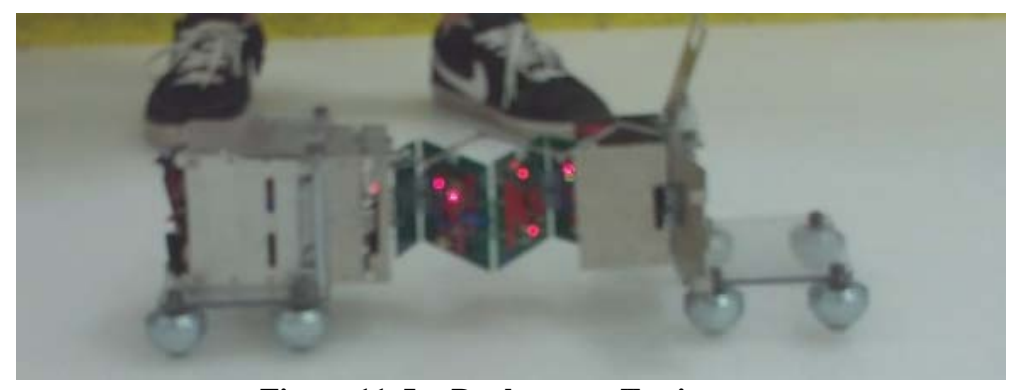

Figure 11. Ice Deployment Testing.

For the timed deployment test, XSAS was installed in the ground testing rig, a device with two platforms with ball rollers mounted to the bottom, and placed on a smooth sheet of ice (see Figure 11). Ice was used to reduce friction as much as possible to simulate, at least in the plane of motion, the microgravity environment. From the video data of the tests, XSAS deployment times were averaged to approximately 0.7 seconds. This value is much lower than what the simulations predicted at approximately four seconds for deployment. The deployment time is taken from when the burn start to the frame in the video that XSAS visibly stopped moving.

Table 1. Ground Deployment Times.

\begin{tabular}{rrrr}
\hline Deployment & & Frames (@ 30 fps) & Time (s) \\
\hline $\mathbf{1}$ & 20 & 0.67 \\
$\mathbf{2}$ & 25 & 0.83 \\
$\mathbf{3}$ & 15 & 0.50 \\
$\mathbf{4}$ & 23 & 0.77 \\
$\mathbf{5}$ & 17 & 0.57 \\
$\mathbf{6}$ & 23 & 0.77 \\
$\mathbf{7}$ & 25 & 0.83 \\
Average Time (s) & & 0.70 \\
\hline
\end{tabular}


Resonance frequency tests were also conducted on the XSAS flight payloads. These data are important for understanding possible interactions between XSAS and the rest of the spacecraft while on orbit. The test procedure included clamping down the payload in its fully deployed configuration and letting the structure hang down in a vertical position. XSAS was then twisted to a slight degree and released. Once calibrated, IMU's located on the upper and lower XSAS assemblies collected gyroscopic data from the resulting motion. From these tests, it was found that XSAS has an average resonance frequency of $4.95 \mathrm{~Hz}$. A characteristic plot of the resonance frequency is shown in Figure 12.

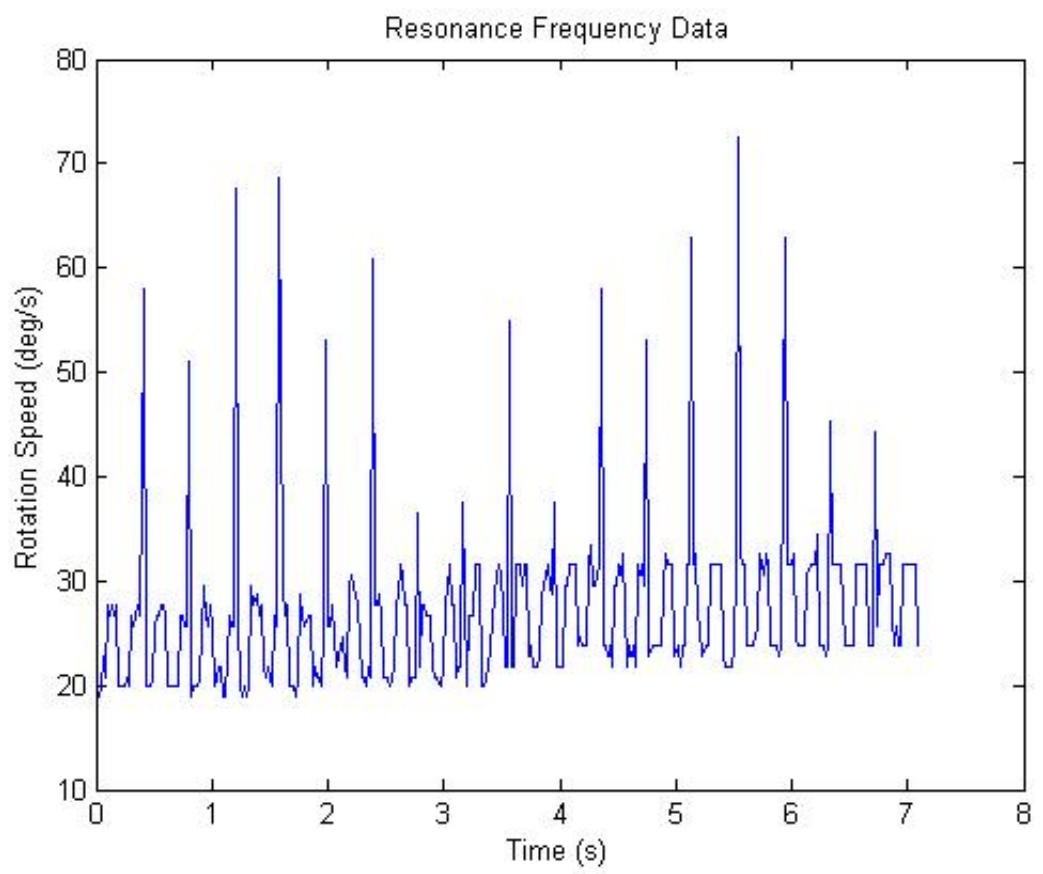

Figure 12. Characteristic plot of the resonance frequency of XSAS. The fundamental mode of the XSAS structure is at $4.95 \mathrm{~Hz}$.

An important part to designing and building XSAS is ensuring performance in the harsh thermal environment of space. Special attention was brought to the hinges as they are not space rated and the pin and hinge portion are made out of the same material, hence have the same coefficient of thermal expansion. Thermal testing of the hinges was completed to insure that a large temperature change wouldn't allow the material to expand so much that the hinge would seize. The test took place inside an oven with a prototype model of the scissor structure and panel/hinge set up as shown in Figure 13.

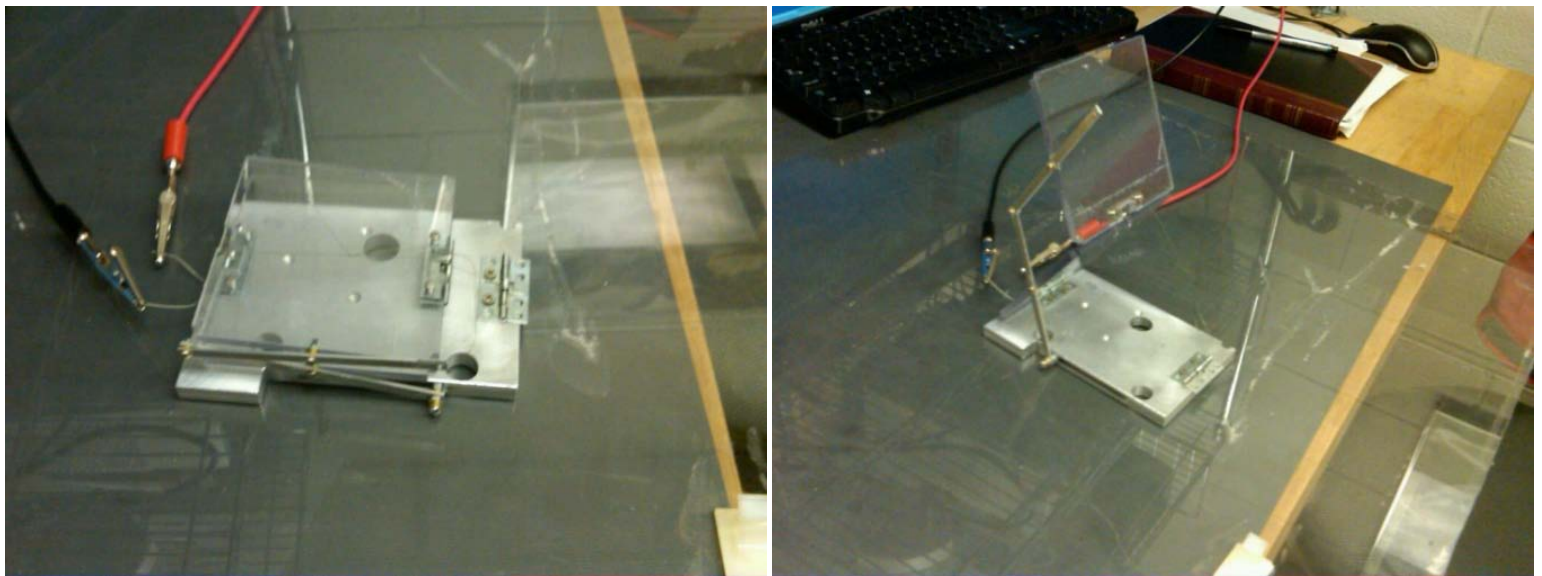

Figure 13. Scissor Structure Prototype Rig.

The prototype rig took approximately one hour to reach $100^{\circ} \mathrm{C}$ once the oven was turned on. Scissor deployment occurred approximately three seconds after the power supply was turned on. There was no noticeable difference in the smoothness of deployment as compared with previous deployments outside of the oven. This leads to the conclusion that thermal expansion of the hinges and scissor does not affect or impinge 
deployment. Additionally, since the test was performed at $100^{\circ} \mathrm{C}$ there should be no concern for thermal expansion affecting deployment on orbit since the maximum expected temperature would be less than approximately $80^{\circ} \mathrm{C}$. Future tests will examine the potential for cold-welding and other unique environmental challenges.

\section{B. Flight Tests}

Microgravity flights provide a unique opportunity to test the XSAS structure in microgravity without having to place it on orbit. These flights, which stretched over two days, had a total of 60 microgravity periods with each lasting roughly 18-20 seconds. Of the 60 microgravity periods, data was captured during 41 attempts. The main source of data was captured through video cameras facing the front and side of the CubeSat as shown in Figure 14. To synchronize the video data with the data captured through the Command and Data Handling system, LED's were placed on the $1 \mathrm{U}$ CubeSat bus that blinked different colors for the different stages of deployment (Figure 14).

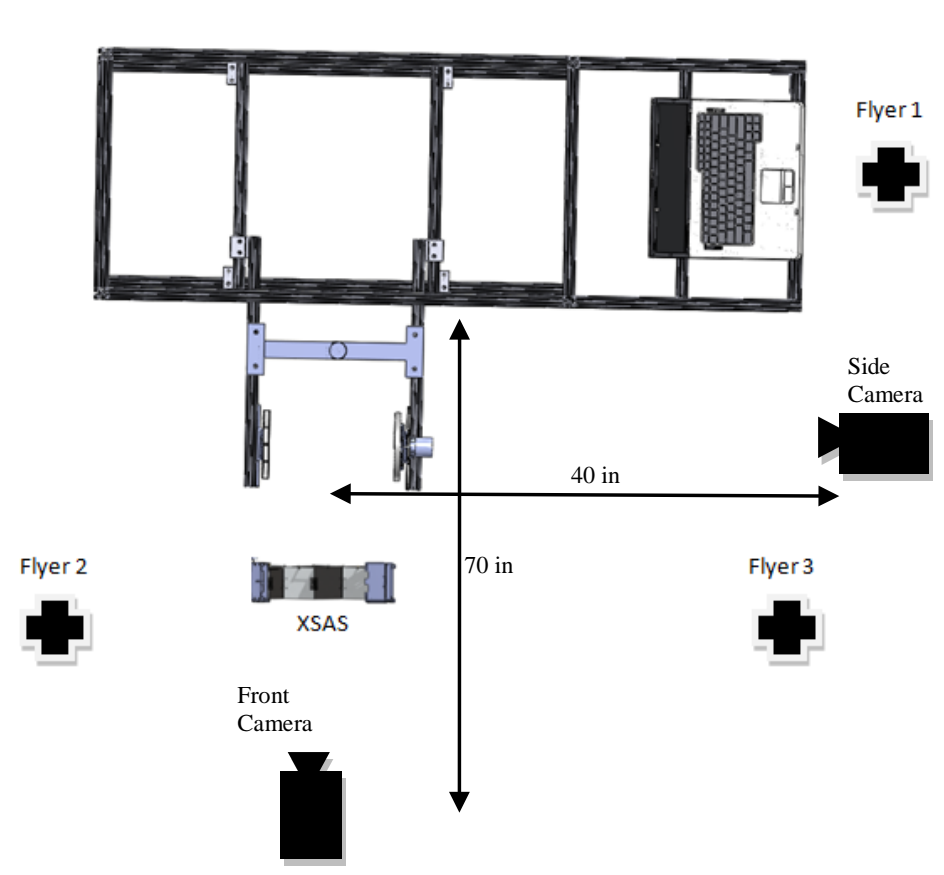

LED Video

Sync Location

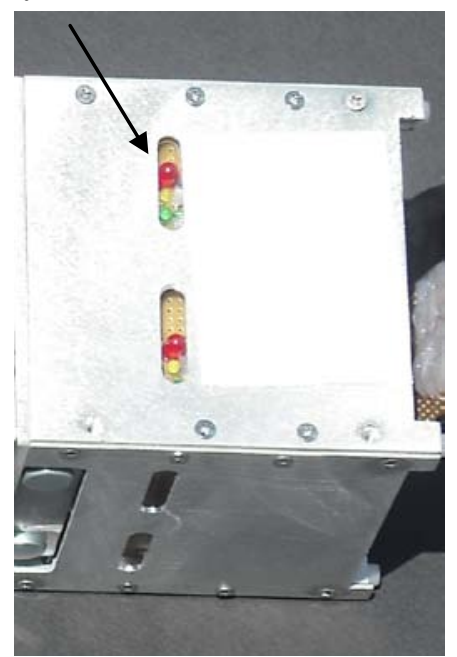

Figure 14. Left: Placement of cameras and flight structure, Right: LED Video Sync System, Red: standby, Red/Green/Yellow: data being logged, Yellow: burn had occurred/currently deploying and Green: latched.

Although much time was spent designing a test set up to rotate XSAS at predetermined rates, an unforeseen failure occurred in microgravity precluding its use. Therefore, XSAS was rotated and released manually. The test duration occurred within approximately ten second windows. Between each microgravity parabola, the panel restraint elastic line would be replaced by a new line for the next parabola.

From the video data, information can be collected about rotation rates, deployment times, and visible bounce back upon latching. The front view camera was placed 70 inches away, and the side view camera was 40 inches away from the center of the flight structure. Data from the cameras were then compared to the calibrated IMU data. Table 2 shows available deployment times and Table 3 shows the rotation speeds for each deployment. On average, XSAS deployed in 0.43 seconds and slowed down $56 \mathrm{deg} / \mathrm{s}$ after deployment. 
Table 2. XSAS deployment times captured on video.

\begin{tabular}{cccccc}
\hline \multicolumn{2}{c}{ Day 1: Deployment Time (s) } & \multicolumn{3}{c}{ Day 2: Deployment Time (s) } \\
\hline Parabola & Front & Side & Parabola & Front & Side \\
5 & 0.34 & NC & 2 & 0.4 & NC \\
6 & 0.37 & NC & 5 & 0.37 & NC \\
7 & 0.3 & NC & 6 & 0.33 & NC \\
9 & 0.33 & NC & 8 & NC & 0.43 \\
10 & 0.37 & 0.8 & 16 & 0.43 & 0.43 \\
18 & 0.5 & 0.5 & 17 & 0.4 & 0.43 \\
21 & 0.4 & 0.4 & 18 & 0.43 & NC \\
22 & 0.36 & 0.36 & 19 & 0.5 & NC \\
Average & $\mathbf{0 . 3 7}$ & $\mathbf{0 . 5 1}$ & & $\mathbf{0 . 4 1}$ & $\mathbf{0 . 4 3}$ \\
\multicolumn{7}{l}{ Average Deployment Time : } \\
\hline \multicolumn{7}{r}{ NC: Not captured, either XSAS was out of the frame or it was too out of focus to tell }
\end{tabular}

Table 3. Average Rotation Rates for both flight days.

\begin{tabular}{cccc}
\hline \multicolumn{4}{c}{ Day 1 } \\
\hline $\begin{array}{c}\text { Parabola } \\
\#\end{array}$ & $\begin{array}{c}\text { Before } \\
\text { Deployment } \\
\text { (deg/s) }\end{array}$ & $\begin{array}{c}\text { After } \\
\text { Deployment } \\
\text { (deg/s) }\end{array}$ & Difference \\
$\mathbf{5}$ & 257 & 98 & 98 \\
$\mathbf{6}$ & 469 & 129 & 129 \\
$\mathbf{7}$ & 363 & 180 & 180 \\
$\mathbf{9}$ & 168 & 8 & 8 \\
$\mathbf{1 0}$ & 153 & 50 & 50 \\
$\mathbf{1 8}$ & 63 & 19 & 19 \\
$\mathbf{2 1}$ & 120 & 46 & 46 \\
$\mathbf{2 2}$ & 117 & 31 & 31 \\
\hline & & Day 2 & \\
\hline Parabola & Deployment & Deployment & Difference \\
$\#$ & (deg/s) & (deg/s) & \\
\hline $\mathbf{8}$ & 74 & 51 & 23 \\
$\mathbf{1 6}$ & 76 & 71 & 5 \\
$\mathbf{1 7}$ & 65 & 39 & 26 \\
$\mathbf{1 8}$ & 157 & 63 & 94 \\
$\mathbf{1 9}$ & 100 & 79 & 21 \\
\hline & & & \\
\hline
\end{tabular}

NC: Not captured, either XSAS was out of the frame, was too out of focus to tell, or the data was too noisy.

As noted above, the average time to deploy and latch XSAS in microgravity was 0.43 seconds. This deployment time is shorter than what had been seen in ground testing by 0.3 seconds. This large difference may come from the friction associated with the ground-based test rig (between the coasters and the ice) as well as added friction in the structure from gravity (between moving parts in the array).

Due to post processing calibration issues and damage to the strain gauges, accurate measurements of strain on the XSAS panels in microgravity are not available. However, the data show that the largest strains occurred as XSAS reached latching. Figure 15 shows a frame by frame shot of what happens from initial deployment to latching. During deployment several phenomena were noticed. First, XSAS deployed linearly, but the top 
assembly did not deploy straight out and would tilt to one side on all the deployments. This has the potential to damage solar cells, because the skirt contacts the solar panel array. This problem was noticed during ground tests and the torsion spring was taken out of the hinge. Although this partially fixed the problem, the upper assembly still tended to tilt to one side rather than deploying straight. This issue is currently undergoing redesign.

From the video data, there was some visible bounce back in the structure at the end of deployment. This bounce back is partially due to the fast deployment speeds. Future designs must address this challenge by adding a damping system to slow down deployment. This bounce back could also be caused by the latching mechanism. The latching mechanism has some extra room, that when deployed at fast speeds it could deform for a small amount of time before resettling into its resting configuration. This causes extra strain on the latching mechanism and parts that go with it. However, this latching mechanism will not be the one used for the final configuration of XSAS. It was redesigned to be easily resettable and thus lacks some of the strength of the original latching mechanism. This problem shouldn't be apparent in the final model since the original latching mechanism is much stronger and does not allow for bending which would cause the structure to extend past its final resting position.

As predicted, the rotation speed slowed down consistently after deployment. This is a function of the four release panels changing the moment of inertia of the system. Although predicted that XSAS would have a resting resonance frequency, the time in microgravity did not allow for that data to be collected after deployment. In most instances XSAS had a clean deployment but would collide with the flight structure or the floor or be caught by a flyer immediately after deployment.

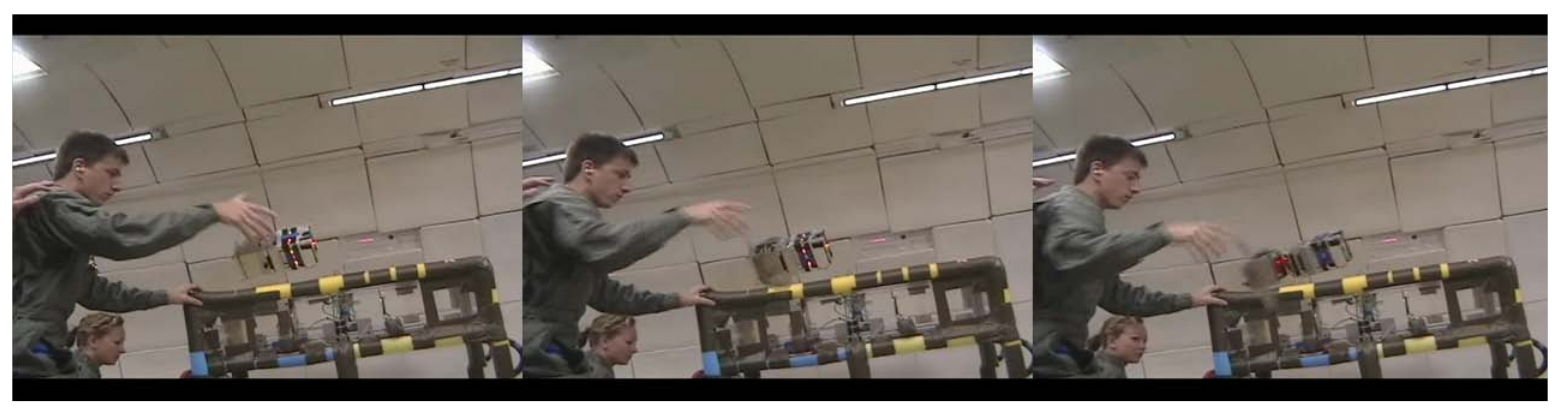

Burn signal sent

Deployment Starts

0.08 seconds after deployment

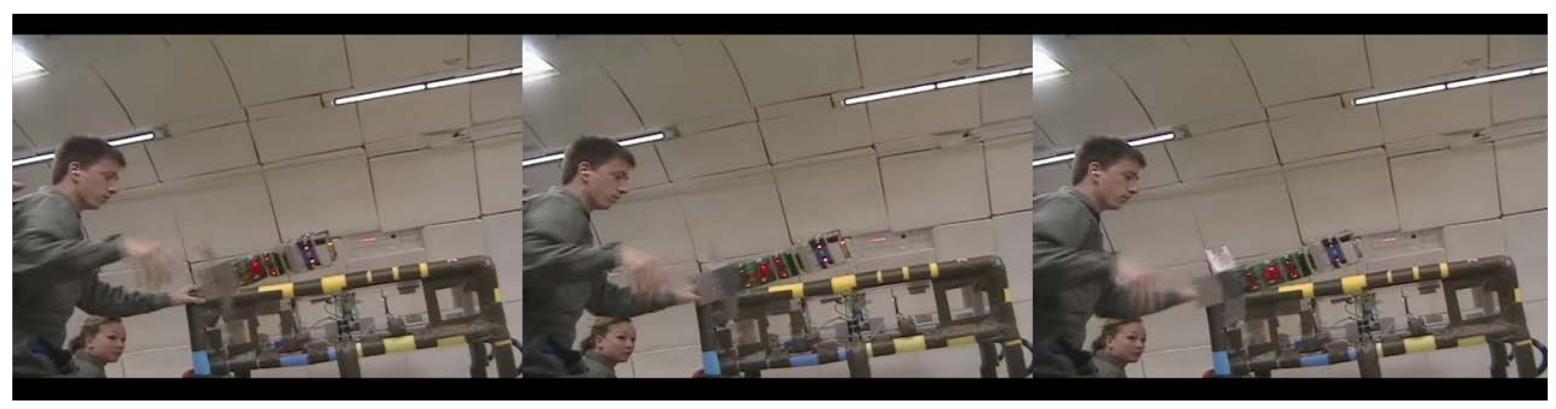

0.16 seconds after deployment

0.2 seconds after deployment

0.24 seconds after deployment

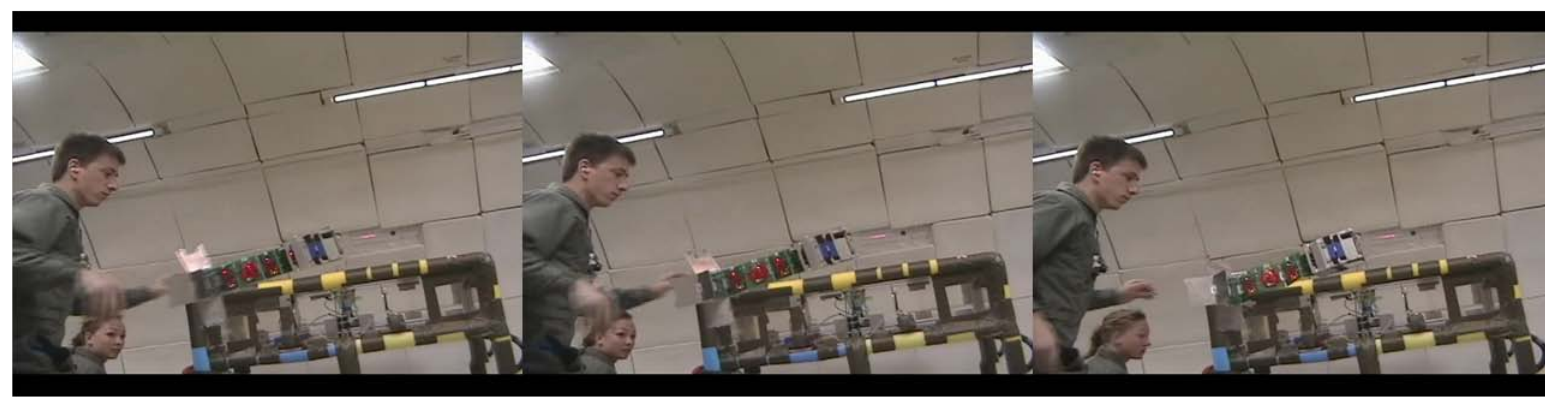

0.28 seconds after deployment

0.32 seconds after deployment

0.4 seconds after deployment

Figure 15. XSAS microgravity deployment sequence. 


\section{Conclusion}

There have been several lessons learned from the microgravity testing of XSAS that can be applied to other teams developing deployable structures. A great deal of time must be spent in design and prototyping the payload and structure. Rapid prototyping is highly recommended to test out the idea in a quick fashion to ensure feasibility. The more ground testing that can be done before a microgravity flight the better. The earlier testing can begin before flight, the more successful and accurate the data will be.

Testing any device in microgravity and developing the platform for it is very difficult due to the nature of the environment. Most researchers have a vague grasp of this environment, but until the device is actually flown, there is no way to know what exactly will happen. In the case of the flight structure, it was assumed that XSAS would not stick to the release panels and that it would deploy roughly in the same position it was released. Both assumptions were wrong. Also, microgravity testing environments may not provide consistent 0g testing conditions, which should be taken into consideration when designing experimental procedures. Most of the video data that capture XSAS was able to only capture parts of deployment but not the whole sequence. As for the flight structure, the amount of friction that the legs of XSAS would have with the rotation structure was underestimated, and this affected the ability to reset it in microgravity.

Deployable structures by their nature are complicated mechanisms. Unforeseen rotations can be induced from the deployment process, so symmetric deployment, or translational (rather than rotational) deployment may be necessary to assure minimal disturbances. The XSAS microgravity testing has shown that oscillations can occur through flexing of the structure when extreme forces are encountered. Thus, a damping system may be required for particularly massive structures to avoid these stresses. Furthermore, ground testing with reduced friction still did not show the speed at which XSAS deployed in microgravity.

From the valuable data gained from prototyping, building, and testing XSAS in microgravity the next iteration of XSAS can begin. This project has taken deployable testing to the next level by adding the next step to testing it in a space like environment. Observations were taken of the actual deployment characteristics of XSAS and potential problems that deployment with this design could cause if flown on an actual mission. These observations and dynamics can be applied to many booms that share similar structural characteristics of XSAS. As this array technology continues in its design iterations, more observations will be made for CubeSat arrays of this nature to ensure the success of future deployable designs. Future work will include a design iteration of XSAS and additional testing in the lab and in microgravity environments.

\section{Acknowledgments}

The authors would like to thank the following programs and people who supported the XSAS program: Prof. Wei Shyy and the Aerospace Engineering Department at Michigan for providing substantial financial and technical support, Bonnie Bryant and the Michigan Space Grant Consortium for supporting both the project and its extensive outreach program, Dean Munson and the University of Michigan for providing substation financial support, the University of Michigan Engineering Council for contributing to the success of our project, the Wilson Center Staff who greatly assisted the design process, the Student Space Systems Fabrication Laboratory for providing a place to work and a great deal of aid, the Reduced Gravity Program at NASA for providing the opportunity for the XSAS microgravity testing, Kiko Dontchev and Theresa Beihle for providing wonderful advice and supporting the next generation of NanoSat Pipeline students, the lab of Prof Perkins for assisting with data analysis and IMU instruction, Arun Kumar and Ken Becker for their help in debugging the electronics system, and finally, we would like to thank all the students and professors who transformed XSAS from a paper design to a flight-tested vehicle.

\section{References}

${ }^{1}$ Silver M., Dobson B., Warren P., "Development of a Deployable Gravity Gradient Boom CubeSat”, 2009 CubSat Developers Workshop, Cal Poly, San Luis Obispo, CA, 2009.

${ }^{2}$ Long M., "A CubeSat Derived Design for a Unique Academic Research Mission in Earthquake Signature Detection," $16^{\text {th }}$ Annual/USU Conference on Small Satellites, SSC02-IX-6, Logan, Utah, 2002.

${ }^{3}$ Bouwmeester J., "Preliminary mission results and project evaluation of Delfi-C ${ }^{3}$ Nano-Satellite",

${ }^{4}$ Thomas, G. "Prototype Development and Dynamic Characterization of Deployable CubeSat Booms” M.S., Aeronautics and Astronautics Dept., Air Univ., Wright-Patterson Air Force Base, OH, 2010.

${ }^{5}$ Hoyt R., Slostad J., “The Multi-Application Survivable Tether (MAST) Experiement” AIAA-2003-5219, 2003.

${ }^{6}$ Bedford, A., Fowler, W. Engineering Mechanics: Dynamics. Prentice Hall, 2007. Print.

${ }^{7}$ Bedford, A., Liechti, K. Mechanics of Materials. Prentice Hall, 2000. Print.

${ }^{8}$ Lan, W. "Poly Picosatellite Orbital Deployer Mk III ICD." The CubeSat Program. California Polytechnic State University, San Luis Obispo, California, Aug. 2, 2007.

${ }^{9}$ Lee, S., Hutputanasin, A., Toorian, A., Wenschel, L., Manukata, R. “CubeSat Design Specification.” The CubeSat Program. California Polytechnic State University, San Luis Obispo, California, Aug. 1, 2009. 
${ }^{10}$ Wertz, J., Larson, Wiley, et al. Space Mission Analysis and Design. Microcosm Press: Hawthorne, CA and Springer: New York, NY, 1999. Print.

${ }^{11}$ Senatore P., "Concept, Design, and Prototyping of XSAS: A High Power Extendable Solar Array for CubeSat Applications”, Proceedings of the $40^{\text {th }}$ Aerospace Mechanisms Symposium, CP-2010-216272, NASA, May 12, 2010, pp 431-444 